\title{
Isolated carbapenem resistant bacteria, their multidrug resistant profile, percentage of healthcare associated infection and associated mortality, in hospitalized patients in a University Hospital in Bratislava
}

\author{
Jalali Y, Sturdik I, Jalali M, Payer J \\ 5th Department of Internal Medicine, University Hospital Bratislava, Bratislava, Slovakia. \\ jalali_yashar@yahoo.com
}

\begin{abstract}
OBJECTIVES: The absence of clinical studies in Slovakia on carbapenem-resistant $K$. pneumoniae, $A$. baumannii, and $P$. aeruginosa, makes planning and treatment strategies challenging and less effective. Our aim is to provide new clinical data on the percentage of healthcare-associated infection, antibiotic resistance profile, and mortality risk associated with these carbapenem-resistant bacteria in our department.

METHODS: An observational, prospective, cross-sectional study was performed for a period of 15 months. Strain identification was performed after classical isolation and bio-chemical and cultivation methods. Antibiotic testing results were interpreted according to EUCAST guideline.

RESULT: Associated mortality risk was the highest in patients diagnosed with $A$. baumannii. The urinary tract was the most common site of isolation in all the three types of bacteria. Colistin was shown to be the most potent antibiotic among all the three types of bacteria, followed by aminoglycoside for $K$. pneumoniae and $P$. aeruginosa and ampicillin-sulbactam for $A$. baumannii.

CONCLUSION: A. baumannii poses the biggest challenge in the treatment and management of infected patients in our centre in Bratislava. Cephalosporins of the second to fourth generation, quinolones, tetracyclines, and cotrimoxazole are widely ineffective in the treatment of isolated bacteria. Reintroduction of colistin, despite its drug toxicity, can be considered as the last resort treatment I (Tab. 4, Fig. 4, Ref. 30). Text in PDF www.elis.sk

KEY WORDS: carbapenem resistant, ESKAPE bacteria, multidrug resistant, healthcare-associated infection.
\end{abstract}

\section{Introduction}

Antibiotic misuse and evolutionary process have contributed to an increase of antibiotic resistance (ABR) worldwide (1). Every year, ABR adds a 1.1 to 1.5 billion-euro burden to healthcare expenses in the European Economic Area (2). By 2050, ABR is predicted to cause a 1.1 to $3.8 \%$ loss in global gross domestic product (3). The World Health Organization (WHO) prepared a list of ABR organisms that pose the greatest threat to humans and should be a priority for research and development of new antibiotics (4). The list includes a group of bacteria, collectively known as ESKAPE, consisting of Escherichia coli, Staphylococcus aureus, Klebsiella pneumoniae, Acinetobacter baumannii, Pseudomonas aeruginosa, and Enterococcus spp., known for their ability to adapt to new environmental challenges and use of different mechanisms to

5th Department of Internal Medicine, University Hospital Bratislava, Bratislava, Slovakia

Address for correspondence: Y. Jalali, MD, 5th Department of Internal Medicine, University Hospital Bratislava, Ružinovská 4810/6, SK-821 01 Bratislava, Slovakia.

Phone: +421.944944520 "escape" antibiotics (4). In recent years, these microorganisms are increasing the cause of infectious diseases in humans. The WHO categorized the need for new antibiotics in three priority classes: critical, high, and medium (4). The carbapenem resistant (CR) A. baumannii, P. aeruginosa, K. pneumoniae and Enterobacter spp. are listed in the critical priority category (4). Focusing attention on priority category bacteria and raising awareness in healthcare providers can reduce the incidence of infected patients receiving inappropriate antibiotic therapy, thus reducing the rates of mortality and transmission. The resistance rate of the critical pathogens has been shown to differ across Europe, likely due to different levels of antibiotic use $(5,6)$.

The recent study showed that CR K. pneumoniae is the fastest growing ABR threat in terms of mortality and morbidity in Europe (7). One of the reasons for this bacteria's increase is caused by the acquisition of carbapenemase enzymes that can hydrolyse carbapenems (the last line antibiotic used for the treatment of multi-resistant bacteria) and other $\beta$-lactam antibiotics to different degrees (8). The genes responsible for producing carbapenemase enzymes are associated with mobile elements that can spread horizontally within and between bacterial species $(7,9)$. Further, K. pneumoniae has been identified as the crucial point of entry 


\section{9-385}

of $\mathrm{ABR}$ genes in the Enterobacteriaceae family $(10,11)$.

ABR mechanisms are targeting both the first line and broad-spectrum agents; in A. baumannii, carbapenem and multi-drug resistance has nearly doubled over the past decade (12). This bacterium has emerged as the most important nosocomial pathogen causing lethal infections in intensive care units according to the study in 2017 (13). A. baumannii is commonly identified in ventilator-associated lung infections, wound infections, urinary tract infections, and meningitis (14). Because it is difficult to eradicate A. baumannii from hospital environments, this bacterium persists on surfaces for a long time and frequently causes nosocomial outbreaks with a high rate mortality (15). Besides predisposition to acquiring $\mathrm{ABR}$ genes, this bacterium has a high intrinsic resistance to a number of agents, contributing to the rise of new pathogens that are more complex and difficult to treat (16).

The intrinsic non-susceptibility of $P$. aeruginosa to many commonly used antibiotics and its ability to survive in various environments has caused an increase in the rate of multi-drug resistant (MDR) healthcare-associated infections (HAI) involving this agent $(17,18)$. The recent study showed that bloodstream infections of $P$. aeruginosa had a higher mortality rate compared to infections caused by bacteria in the Enterobacteriaceae family (19). The primary mechanism for their ABR is attributed to chromosomal mutations that produce altered prions, change in efflux pump activity, and intrinsic production of $\beta$-lactamases (18). Carbapenemase genes, carried on mobile elements like in K. pneumoniae, are the mechanism for gaining resistance to carbapenems and have a potential for rapid dissemination (18). In the study in 14 European countries, the prevalence of metallo- $\beta$ lactamase (MBL)-producing P. aeruginosa showed an increase from $12.3 \%$ in 2010 to $30.6 \%$ in 2011 (20).

The objectives of this study were to determine the number of isolated cases of carbapenem-resistant, critical priority ESKAPE bacteria (namely, K. pneumoniae, A. baumannii, and P. aeruginosa) in hospitalized patients, record the age and gender demography of infected patients, and collect information about the relation of hospitalization duration to healthcare-associated infection, antibiotic resistance profile, associated mortality risk and the most frequent isolation sites for each bacterium.
Tab. 1. Gender and age of patients, hospitalization duration before infection diagnosis, and deaths in relation to MDR and HAI.

\begin{tabular}{|c|c|c|c|c|}
\hline Total No of cases & & & 48 cases & Cases $(\%)$ \\
\hline Total No of diagnosed patients & & & 44 cases & \\
\hline No of male patients & & & 27 cases & $61 \%$ \\
\hline Age of male patients & & $(50-95 \mathrm{Y})$ & Mean $72 \mathrm{Y}$ & \\
\hline No of female patients & & & 17 cases & $39 \%$ \\
\hline Age of female patients & & $(29-92$ Y) & Mean $60 \mathrm{Y}$ & \\
\hline Hospitalization duration (days) & Min .1 & Max. 68 & Av. 34.5 & \\
\hline $\begin{array}{l}\text { Hospitalization duration (days) } \\
\text { before diagnosis with HAI + MDR }\end{array}$ & Min. 3 & Max. 44 & Av. 23.5 & \\
\hline Total No of deaths & & & 14 cases & $32 \%$ \\
\hline No of deaths in males & & & 11 cases & $79 \%$ \\
\hline No of HAI to NHAI deaths in males & HAI 7 & NHAI 4 & & $64 \% / 36 \%$ \\
\hline No of HAI + MDR deaths in males & & & 6 cases & $54 \%$ \\
\hline No of deaths in females & & & 3 cases & $21 \%$ \\
\hline No of HAI to NHAI deaths in females & HAI 3 & NHAI 0 & & $100 \% / 0 \%$ \\
\hline No of HAI + MDR deaths in females & & & 2 cases & $67 \%$ \\
\hline
\end{tabular}
diagnosis with MDR + HAI in death cases

HAI: Healthcare-associated infection, NHAI: Non-healthcare associated infection, MDR: Multidrug resistant bacteria (in this study, all MDR bacteria are also CR by default), CR: Carbapenem resistant bacteria (but not multidrug resistant), Av.: Average, Min.: minimum, Max.: Maximum, M: Male, F: Female

Tab. 2. Number of MDR and HAI cases and their relation to each other and to hospitalization duration and death count for each bacterium.

\begin{tabular}{|c|c|c|c|c|c|}
\hline & No & $\begin{array}{c}\text { Cases } \\
(\%)\end{array}$ & $\begin{array}{l}\text { Av. hospit. } \\
\text { duration (days) } \\
\text { until diagnosis }\end{array}$ & $\begin{array}{l}\text { No of } \\
\text { deaths }\end{array}$ & $\begin{array}{c}\text { Deaths } \\
(\%)\end{array}$ \\
\hline \multicolumn{6}{|l|}{ K. pneumoniae } \\
\hline Total No of cases & 14 & & & 4 & $29 \%$ \\
\hline No of MDR cases & 9 & $64 \%$ & 22 & 3 & $33 \%$ \\
\hline No of HAI cases & 8 & $57 \%$ & 21 & 3 & $43 \%$ \\
\hline No of NHAI cases & 6 & $42 \%$ & & 1 & \\
\hline No of MDR cases among HAI cases & 6 & $86 \%$ & 26 & 3 & $50 \%$ \\
\hline \multicolumn{6}{|l|}{ P. aeruginosa } \\
\hline Total No of cases & 16 & & & 4 & $25 \%$ \\
\hline No of MDR cases & 11 & $69 \%$ & 6 & 4 & $36 \%$ \\
\hline No of HAI cases & 9 & $56 \%$ & 11 & 1 & $11 \%$ \\
\hline No of NHAI cases & 7 & $44 \%$ & & 3 & \\
\hline No of MDR cases among HAI cases & 7 & $78 \%$ & 9 & 1 & $14 \%$ \\
\hline \multicolumn{6}{|l|}{ A. baumannii } \\
\hline Total number of cases & 18 & & & 8 & $44 \%$ \\
\hline No of MDR cases & 16 & $89 \%$ & 16 & 7 & $44 \%$ \\
\hline No of HAI cases & 18 & $100 \%$ & 14 & 8 & $44 \%$ \\
\hline No of NHAI cases & 0 & $0 \%$ & & 0 & \\
\hline No of MDR cases among HAI cases & 16 & $89 \%$ & 16 & 7 & $44 \%$ \\
\hline
\end{tabular}

HAI: Healthcare-associated infection (an infection that patient acquires after minimum of 48 hours hospitalization with which has was not diagnosed before), NHAI: Non-healthcare associated infection (infection which patient already acquired before being hospitalized), MDR: Multidrug-resistant bacteria (in this study all MDR bacteria are as well CR by default), Av.: Average, No: Number, Hospit.: Hospitalization 


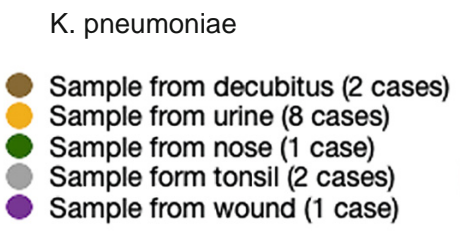

P. aeruginosa

Sample from decubitus (1 case) Sample from urine (7 cases) Sample from nose (3 cases) Sample from wound (3 cases) Sample from sputum (2 cases)

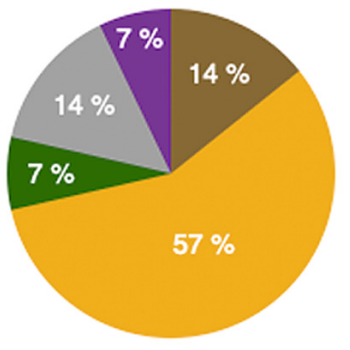

\section{A. baumannii}

Sample from decubitus (2 cases) Sample from urine (5 cases) Sample from nose (1 case) Sample form tonsil (3 cases) Sample from wound (5 cases) Haemoculture (2 cases)
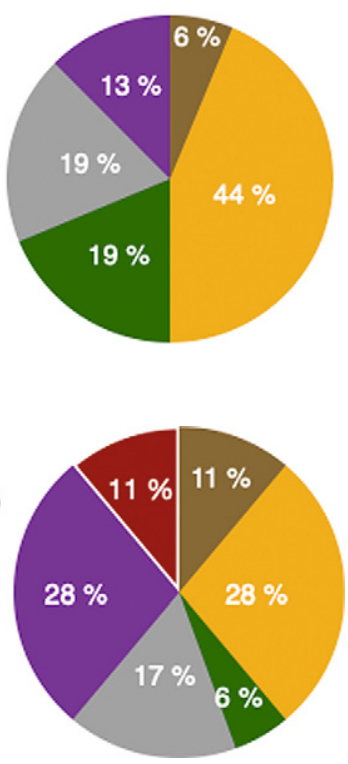

Fig. 1. Percent of diagnosed cases by sampling site for each bacterium.

collected information on the following variables: the causative bacterial agent, place of sampling, antibiogram, whether it was a HAI or non-healthcare associated infection (NHAI), whether the patient was dismissed or diseased during our monitoring, duration of the present hospitalization, and duration of hospitalizations before diagnosis of the CR case. There was no specific process for the selection of patients in this study; sampling and cultivation examination was performed on patients based on the treatment method chosen by the patient's doctor. However, all the patients included in study had active infectious processes with raised inflammatory markers.

Sterile swabs were used to collect samples from the nose, tonsils, wounds, and decubitus. Blood sampling was performed using peripheral intravenous puncture. Urine was collected by mid-stream urine sampling or via urinary catheter. Samples were processed through conventional methods approved by Slovak ministry of health. Strain identification was performed after the classical isolation and classical bio-chemical and cultivation methods by the Bruker
MALDI Biotyper (Bruker, Massachusetts, USA), which uses the MALDI-TOF Mass Spectrometry method (21). Antibiotic testing results were interpreted according to EUCAST guideline (European Committee on Antimicrobial Susceptibility Testing) (22).

Data collected during the monitoring period were analysed using Microsoft Excel and Analyse-it software. We used the twosided Fisher's exact test for calculating the $p$ value ( $\mathrm{p}$ value was set as $\mathrm{p} \leq 0.05$ ).

Ethical review and approval were not required for the study (as an observational study) on human participants in accordance with local legislation and institutional requirements. Written informed consent for participation was not required for this study in accordance with national legislation and the institutional requirements.

\section{Results}

In our study, we diagnosed 48 cases of ESKAPE carbapenemresistant bacteria from 44 (in 2 patients we isolated all three types of bacteria) patients hospitalized in our clinic (27 male patients and 17 female patients) (Tab. 1). None of the patients underwent surgeries or invasive procedures prior to their admission to therapy.

MDR was defined according to the European Centre of Disease Control criteria as "acquired non-susceptibility to at least one agent in three or more antimicrobial categories" (23).

We found 14 cases of carbapenem-resistant K. pneumoniae, nine of which were MDR ( $64 \%$ of cases). Six patients out of the 9 MDR cases ( $86 \%$ of cases) were found to be HAI. The six patients were hospitalized for the average of 26 days before being diagnosed and half of them passed away during hospitalization (Tab. 2). We found 16 cases of $P$. aeruginosa, 11 of which were MDR cases (69\% of cases). Seven patients out of 11 MDR cases were HAI ( $78 \%$ of cases). The seven patients were hospitalized for the average of nine days before being diagnosed. One of the seven patients passed away during hospitalization (Tab. 2). We found 18 cases of A. baumannii, 16 of which were MDR cases ( $89 \%$ of all cases). All the MDR cases were HAI. The 16 patients were hospitalized for the average of 16 days before being diagnosed; 44 $\%$ of these patients passed away during hospitalization (Tab. 2). This is highest mortality (regardless of whether the bacterium was the causative agent) recorded among all three types of bacteria.
Tab. 3. Resistance of isolated bacteria to various types of antibiotics.

\begin{tabular}{|c|c|c|c|}
\hline Antibiotic agents & $\begin{array}{c}\text { K. pneumoniae } \\
\text { Resistance } \mathrm{n} / \mathrm{N} \%\end{array}$ & $\begin{array}{c}\text { A. baumannii } \\
\text { Resistance } n / \mathrm{N} \%\end{array}$ & $\begin{array}{c}\text { P. aeruginosa } \\
\text { Resistance } n / \mathrm{N} \%\end{array}$ \\
\hline Ampicillin - sulbactam & (12/14) $85.7 \%$ & (2/18) $11.1 \%$ & (16/16) 100.0\% \\
\hline Tazocin & $(11 / 14) \quad 78.5 \%$ & (17/18) 94.4\% & $(14 / 16) \quad 87.5 \%$ \\
\hline Cefuroxime & (11/14) $78.5 \%$ & (17/18) 94.4\% & (16/16) $100.0 \%$ \\
\hline Ceftazidime & (12/14) $85.7 \%$ & (17/18) 94.4\% & $(12 / 16) \quad 75.0 \%$ \\
\hline Cefepime & (12/14) $85.7 \%$ & (17/18) 94.4\% & $(14 / 16) \quad 87.5 \%$ \\
\hline Cefoprazone - sulbactam & $(8 / 14) \quad 57.1 \%$ & (16/18) $88.8 \%$ & $(13 / 16) \quad 81.2 \%$ \\
\hline Tobramycin & (9/14) $64.2 \%$ & (7/18) $38.8 \%$ & $(9 / 16) \quad 56.2 \%$ \\
\hline Gentamycin & (9/14) $64.2 \%$ & (7/18) $38.8 \%$ & $(9 / 16) \quad 56.2 \%$ \\
\hline Amikacin & (5/14) $35.7 \%$ & (6/18) $33.3 \%$ & $(8 / 16) \quad 50.0 \%$ \\
\hline Colistin & $(1 / 14) \quad 7.1 \%$ & $(0 / 18) \quad 0.0 \%$ & $(2 / 16) \quad 12.5 \%$ \\
\hline Ciprofloxacin & (10/14) $71.4 \%$ & (17/18) $94.4 \%$ & $(12 / 16) \quad 75.0 \%$ \\
\hline Tetracycline & (10/14) $71.4 \%$ & (17/18) 94.4\% & (15/16) $93.7 \%$ \\
\hline Trimethoprim - sulphamethoxazole & (8/14) $57.1 \%$ & (16/18) $88.8 \%$ & (16/16) $100.0 \%$ \\
\hline
\end{tabular}


Tab. 4. Resistance of different antibiotic types.

\begin{tabular}{lcrc}
\hline Antibiotic / Bacterium & $\begin{array}{c}\text { 1st ATB resistance } \\
\mathrm{n} / \mathrm{N} \%\end{array}$ & $\begin{array}{c}\text { 2nd ATB resistance } \\
\mathrm{n} / \mathrm{N} \%\end{array}$ & p value \\
\hline Cefuroxime/Cefepime in K. pneumoniae & $(11 / 4) 78.5 \%$ & $(12 / 14) 85.7 \%$ & 0.337 \\
Sulprazone/Amikacin in K. pneumoniae & $(8 / 14) 57.1 \%$ & $(5 / 14) 35.7 \%$ & 0.160 \\
Ampicillin-sul./Amikacin in A. baumannii & $(2 / 18) 11.1 \%$ & $(6 / 18) 33.3 \%$ & 0.093 \\
Ceftazidime/Cefepime in P. aeruginosa & $(12 / 16) 75.0 \%$ & $(14 / 16) 87.5 \%$ & 0.241 \\
Colistin/Amikacin in P. aeruginosa & $(2 / 16) 12.5 \%$ & $(8 / 16) 50.0 \%$ & 0.023 \\
\hline
\end{tabular}

ATB: antibiotic

Male patients were more likely to be infected by CR ESKAPE bacteria than female patients ( $61 \%$ to $39 \%$ ). The gender difference in the number of infected patients was most prominent in K. pneumoniae cases, in which males were two and half times as likely to be infected than females (Tab. 2). Although the gender difference was not as significant as in K. pneumoniae, slightly more males were infected with $A$. baumannii and $P$. aeruginosa than females ( $56 \%$ to $44 \%$ in both bacteria). On average, male patients were older than female patients by 12 years (Tab. 1) and thus had more comorbidities at admission, which likely contributed to the significantly longer duration of their hospitalization. Male patients were hospitalized for about twice as long (total $=568$ days, average $=21$ days) as female patients (total $=233$ days, average $=15$ days) and stayed in hospital on average at least a week longer than females. These could initially explain the reason of more prominent male demography among patients (Tab. 1).

In total, 14 patients passed away during hospitalization (11 males and 3 females). It is unknown if the infection was the causative agent for death in the patients in this study as a post-mortem biopsy was not performed on all the patients. However, given the polymorbid profile of most of the patients, who were hospitalized for a long duration due to several complications, and the burden that MDR bacteria can put on the organism, it can be assumed that infection was an important stress factor prior to death. In the patients who died, all but two (86\%) were diagnosed with MDR bacteria.

The average duration of hospitalization until diagnosis of infectious bacteria (including only HAI cases) was the longest in $K$. pneumoniae (average of 21 days) followed by A. baumannii (14 days) and lastly P. aeruginosa (11 days) (Tab. 2). On average, patients caught $\mathrm{CR} K$. pneumoniae after approximately three weeks of hospitalization, CR A. baumannii after two weeks, and CR P. aeruginosa after 11 days. Of course, it is presumable that faster acquisition of these bacterial infections is possible, especially in immunocompromised patients on combined antibiotic therapy.

A similar pattern was observed in the case of MDRs; patients with MDR K. pneumoniae had the longest hospitalization period before being infected and diagnosed ( 22 days on average), followed by A. baumannii (16 days) and P. aeruginosa ( 6 days).

We found that urinary tract infections (UTIs) were the leading source of infection for all the three types of bacteria in our study (Fig. 1). The second most common source of infection for all three types of bacteria was wound and decubitus infections.

Of all the patients diagnosed with UTIs, $63 \%$ of males and $67 \%$ of females were undergoing urinary catheterization during hospitalization. Sixty percent of UTI cases were MDR. Twenty-nine percent of the patients (4 patients), who died during our study had positive UTIs; all of these patients were male and $67 \%$ of them were infected by MDR bacteria (4 out of 6).

In our study, the lowest resistance was recorded for colistin for all the three types of bacteria ( $7 \%$ for K. pneumoniae, $0 \%$ for A. baumannii, and $12 \%$ for $P$. aeruginosa) (Tab. 3). All the three types of bacteria were completely resistant to ampicillin; however, A. baumannii showed a low resistance to ampicillin-sulbactam (11\% resistant). A. baumannii also showed a low resistance to amikacin. The difference in A. baumannii's resistance to ampicillin-sulbactam and amikacin was not statistically significant ( $\mathrm{p}=0.093)($ Tab. 4). K. pneumoniae was highly resistant and $P$. aeruginosa was completely resistant to ampicillin-sulbactam ( $85.7 \%$ and $100 \%$ respectively) (Tab. 3). A. baumannii showed a high resistance to cephalosporins, irrespective of whether it was a second, third or fourth-generation cephalosporin (94.5\% to cefuroxime, $94.5 \%$ to cefotaxime, and $94.5 \%$ to cefepime) (Tab. 3). Conversely, a resistance to third generation cephalosporins was lower than to fourth generation cephalosporins in $P$. aeruginosa and a resistance to second generation cephalosporins was lower than to fourth generation cephalosporins in K. pneumoniae ( $P$. aeruginosa: $75 \%$ for ceftazidime in comparison to $87.5 \%$ for cefepime; K pneumoniae: $78.5 \%$ for cefuroxime in comparison to $85.7 \%$ for cefepime). However, when compared together, the difference between the third- and fourth generation cephalosporins was statistically insignificant (Tab. 4). The lack of statistical significance may be due to the overall increased resistance to all the tested antibiotics.

A resistance to aminoglycosides was generally low in K. pneumoniae and A. baumannii (Tab. 3). Amikacin was the most potent aminoglycoside antibiotic with the lowest resistance $(35.7 \%$ in K. pneumoniae, $33.3 \%$ in A. baumannii) (Tab. 3). However, $P$. aeruginosa was more resistant to aminoglycosides (56.2 \% resistant to gentamycin and tobramycin and $50 \%$ resistant to amikacin). Additionally, $50 \%$ resistance was found to aztreonam in $P$. aeruginosa (same as for amikacin). Overall, resistance of $P$. aeruginosa to colistin was significantly lower than to amikacin $(\mathrm{p}=0.023)($ Tab. 4).

Resistance to macrolides, tetracyclines, and trimethoprimsulphathiazole was high in A. baumannii, and K. pneumoniae. $P$. aeruginosa showed $100 \%$ resistance to trimethoprim-sulphamethoxazole.

As a prerequisite to this study, all the included cases were resistant to carbapenems (Figs 2, 3 and 4).

\section{Discussion}

The predominance of infection by CR ESKAPE bacteria in males (61 \% compared to $39 \%$ in females (Tab. 1), could be partly explained by the age difference between males and females (12 years), which was related to differences in comorbidities, com- 


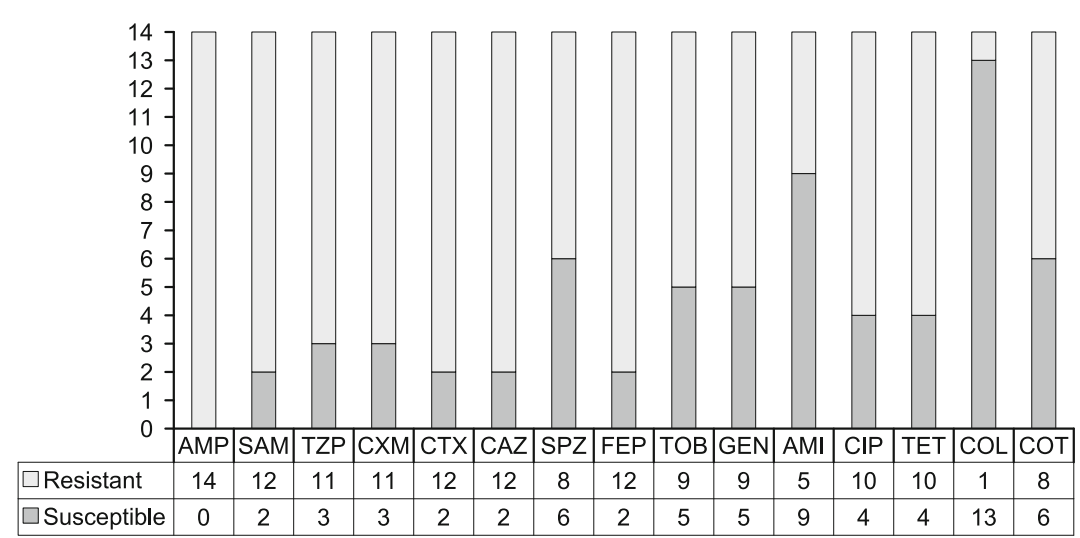

Fig. 2. Antibiotic resistance profile of isolated cases of $K$. pneumoniae included in our study. AMP: ampicillin, SAM: ampicillin-sulbactam, TZP: tazocin (piperacillin-tazobactam), CXM: cefuroxime, CTX: cefotaxime, CAZ: ceftazidime, SPZ: sulperazone (cefoperazone-sulbactam), FEP: cefepime, ATM: aztreonam, TOB: tobramycin, GEN: gentamycin, AMI: amikacin, CIP: ciprofloxacin, TET: tetracycline, COL: colistin, COT: co-trimoxazole (trimethoprim-sulfamethoxazole)

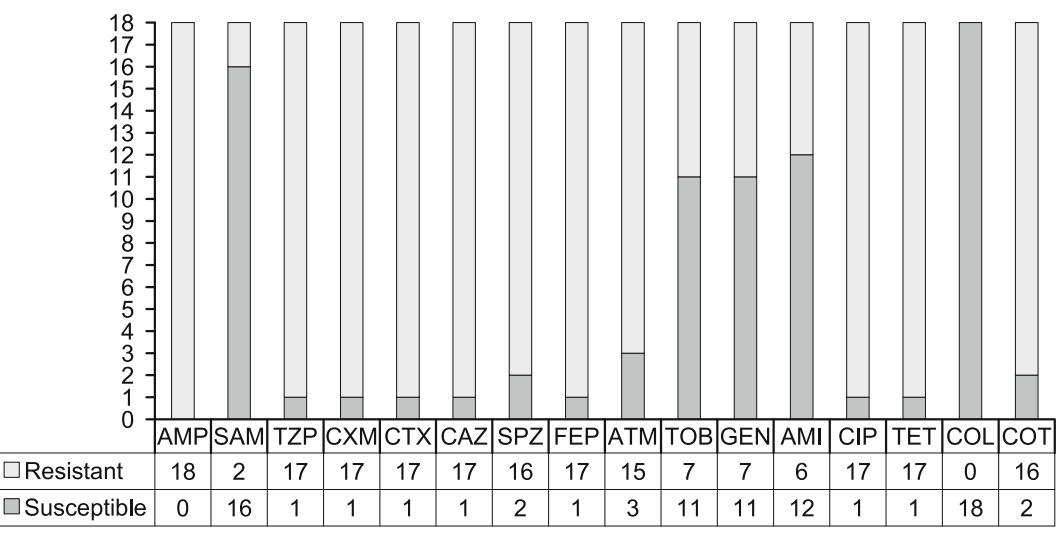

Fig. 3. Antibiotic resistance profile of isolated cases of A. baumannii included our study. AMP: ampicillin, SAM: ampicillin-sulbactam, TZP: tazocin (piperacillin-tazobactam), CXM: cefuroxime, CTX: cefotaxime, CAZ: ceftazidime, SPZ: sulperazone (cefoperazone-sulbactam), FEP: cefepime, ATM: aztreonam, TOB: tobramycin, GEN: gentamycin, AMI: amikacin, CIP: ciprofloxacin, TET: tetracycline, COL: colistin, COT: co-trimoxazole (trimethoprim-sulfamethoxazole).

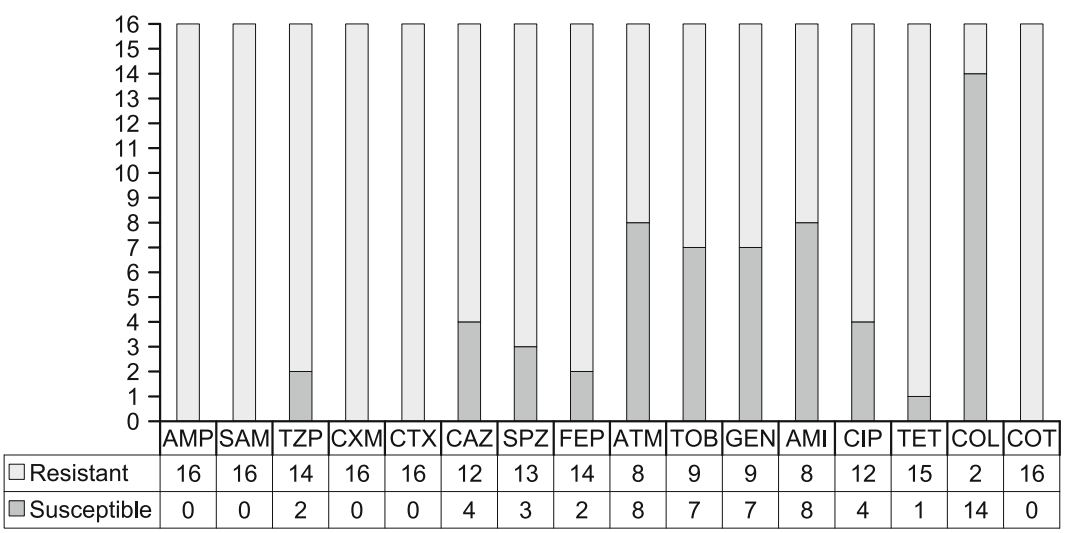

Fig. 4. Antibiotic resistance profile of isolated cases of $P$. aeruginosa included in our study. AMP: ampicillin, SAM: ampicillin-sulbactam, TZP: tazocin (piperacillin-tazobactam), CXM: cefuroxime, CTX: cefotaxime, CAZ: ceftazidime, SPZ: sulperazone (cefoperazone-sulbactam), FEP: cefepime, ATM: aztreonam, TOB: tobramycin, GEN: gentamycin, AMI: amikacin, CIP: ciprofloxacin, TET: tetracycline, COL: colistin, COT: co-trimoxazole (trimethoprim-sulfamethoxazole). plications, and duration of hospitalization. Males were hospitalized on average almost twice as long as females (Tab. 1). This emphasises the association of hospitalization duration to age and comorbidities.

Our results suggest that (our isolated strains of) $P$. aeruginosa requires the shortest hospitalization period before infecting patients (average of 11 days), followed by A. baumannii (14 days), and K. pneumoniae (21 days). This trend is seen in both MDR and CR cases (Tab. 2), which suggests that the longer a patient is hospitalized, the greater the chance of becoming infected by A. baumannii or K. pneumoniae (Tab. 2).

Slovakia is among the European countries with the highest percentage of $\mathrm{ABR}$ over the past years (24).

Based on the ECDC surveillance of antimicrobial resistance in Europe report (24), despite decreasing trends in resistance in the past four years, high resistance percentages and combined resistance persists in many countries, especially in eastern Europe for P. aeruginosa and A. baumannii, including Slovakia. Although the ECDC reports the ABR of each bacterium to a single control antibiotic, it does not report the ABR in carbapenem-resistant strains (as in our study). Nonetheless, we can find similarities between the ABR patterns.

Based on this report, resistance to fluroquinolones, ceftazidime, piperacillintazobactam, and aminoglycosides remains steadily high (with a minor decrease) in Slovakia for P. aeruginosa (24). High resistance to the same antibiotics was seen in isolated $P$. aeruginosa strains in our study (Tab. 3 ). However, the percentage of resistance seems to be much higher in our isolated strains in comparison to the ECDC report. Similarly, high resistance, as expected, is seen among cephalosporins and fluroquinolones in K. pneumoniae (25). Since there has been over a seven-fold increase in CR in K. pneumoniae since 2006 (25), and given that a high number of CR is associated with MDR (25) (64\% of cases in our study), this suggests the need for close continuous monitoring and greater efforts to efficiently respond to this public health threat.

Resistance to aminoglycosides in isolated strains of $A$. baumannii in our study seemed to have a similar pattern as described in the annual resistance report by 


\section{9-385}

the ECDC for Slovakia. However, fluroquinolones are far less potent in our study and isolated strains show considerably more resistance in comparison to the report findings (56.0\% to $94.4 \%$, respectively) (24).

The different pattern of resistance in our study could be partly explained by the low number of cases as the study took place in a single healthcare centre. Further, the study needs to be extended to include additional cases over several years in a multicentre approach in order to provide more complex and sensitive measures of partial utility.

Another limitation of this study is that we did not perform genetic tests to determine the mechanism of resistance to antibiotics.

In our study, colistin was shown to be the most potent antibiotic across all three types of bacteria with the lowest resistance ( $7.1 \%$ in K. pneumoniae, $0 \%$ in A. baumannii, and $12.5 \%$ in $P$. aeruginosa (Tab. 3). Although the use of colistin during the last three decades has been limited due its toxicity, its usefulness is being re-evaluated in studies with multidrug resistance ESKAPE bacteria (26). Despite this, like carbapenems, there are strains among all the three types of bacteria which show a resistance to this last resort antibiotic. In our study, two strains of P. aeruginos $a$ and one strain of $K$. pneumoniae were found to be resistant to colistin (Figs 2 and 4). Several recent studies demonstrated the benefit of drug combination therapy in cases of carbapenem- and colistin-resistant strains of ESKAPE bacteria (26). As an example, the combination of colistin with nicodamids was shown to be an effective treatment of A. baumannii and K. pneumoniae colistinresistant strains (27). The interaction of nicodamids with the negatively charged outer membrane of colistin-resistant strains leads to a synergistic effect with colistin. The combination of colistin with selective oestrogen receptor modulators (SERM) such as tamoxifen, raloxifene, and toremifene, also showed a good activity against polymyxin-resistant strains (28).

However, the question of antibiotic reintroduction or drug repurposing remains rather wide and unanswered. As antibiotics are not part of the chronic treatment regimen, large pharmaceutical companies have low interest in investing in new antibiotics (26). Hence, drug repurposing strategies seem to be a better solution for the time being, although this strategy can also cause adverse interactions. First, drug repurposing and combination can increase toxic side effects, and second, pharmacokinetic, pharmacodynamics, solubility, and conservation considerations among combined drugs can make them incompatible (29). That is why the need for new molecules is more urgent than ever.

\section{Conclusion}

Long-term urinary catherization was the most important predisposing risk factor in hospitalized patients, as UTIs were the most common type of infection among all the three types of bacteria ( $42 \%$ of diagnosed cases) (Fig. 1). Wound and decubitus infections together made up $27 \%$ of diagnosed cases.

A. baumannii was the most frequent isolated bacterium and had the highest number of MDR and associated mortality cases. Since all of the isolated cases were HAI, we can conclude that out of the three bacteria examined in our study, A. baumannii appears to be the most challenging ESKAPE bacteria burdening healthcare in our centre.

Although all the three types of bacteria were completely resistant to ampicillin, ampicillin-sulbactam was still an effective antibiotic for A. baumannii strains and showed a low resistance (11.1\%) (Tab. 4). The recent study from eastern Europe showed a similar sensitivity for this bacterium to this antibiotic (30).

Susceptibility to second to fourth generation cephalosporins was low across all the three types of bacteria. There was no statistically significant difference between the resistance among different generations of cephalosporins in all the three types of bacteria (Tab. 4). Among aminoglycosides, amikacin showed to be the most potent antibiotic with the lowest resistance. Amikacin was the most effective antibiotic after colistin for K. pneumoniae and $P$. aeruginosa and the third most potent antibiotic for A. baumannii.

Colistin proved to be a potent antibiotic for the isolated CR ESKAPE bacteria in our study (Tab. 3). Reintroduction of this antibiotic despite its drug toxicity and complications in MDR cases has been recently re-evaluated (26). Despite that, as in the case of carbapenems, an increasing number of colistin-CR strains are emerging. Several recent studies demonstrated the benefit of drug combination therapy and use of synergy to overcome this problem $(27,28)$. However, the question of antibiotic reintroduction or drug repurposing remains rather unanswered, and the importance of finding new molecules is more urgent than ever.

\section{References}

1. Zhen X, Lundborg CS, Sun X, Hu X, Dong H. Economic burden of antibiotic resistance in ESKAPE organisms: a systematic review. Antimicrobial resistance and infection control 2019; 8: 137.

2. ECDC/EMEA. The bacterial challenge: time to react. ECDC; 2009 17. September, 2009.

3. Jonas OBIA, Berthe FCJ, Le Gall FG, Marquez PV. Drug-resistant infections: a threat to our economic future (Vol 2): final report (English). HNP/Agriculture Global Antimicrobial Resistance Initiative Washington, D.C.: World Bank Group. Working paper 2017 2017/03/01.

4. Organization WH. Global action plan on antimicrobial resistance. WHO; 2015.

5. Pogue JM, Kaye KS, Cohen DA, Marchaim D. Appropriate antimicrobial therapy in the era of multidrug-resistant human pathogens. Clin Microbiol Infect 2015; 21 (4): 302-312.

6. Karlowsky JA, Hoban DJ, Hackel MA, Lob SH, Sahm DF. Antimicrobial susceptibility of Gram-negative ESKAPE pathogens isolated from hospitalized patients with intra-abdominal and urinary tract infections in Asia-Pacific countries: SMART 2013-2015. J Med Microbiol 2017; 66 (1): 61-69.

7. Mathers AJ, Cox HL, Kitchel B, Bonatti H, Brassinga AK, Carroll J et al. Molecular dissection of an outbreak of carbapenem-resistant enterobacteriaceae reveals Intergenus KPC carbapenemase transmission through a promiscuous plasmid. mBio 2011; 2 (6): e00204-002011.

8. David SR, Harris SR, Glasner C, Feltwell T, Argimon S, Abudahab K, Goater R, Giani T, Errico G, Aspbury M, Sjunnebo S, the EuSCAPE Working Group, the ESGEM Study Group, Edward J. Fei, Gian Maria Ros- 
solini, David M. Aanensen, Hajo Grundmann Epidemic of carbapenemresistant Klebsiella pneumoniae in Europe is driven by nosocomial spread. Nature Micorbiology 2019.

9. Martin J, Phan HTT, Findlay J, Stoesser N, Pankhurst L, Navickaite I et al. Covert dissemination of carbapenemase-producing Klebsiella pneumoniae (KPC) in a successfully controlled outbreak: long- and shortread whole-genome sequencing demonstrate multiple genetic modes of transmission. J Antimicrob Chemother 2017; 72 (11): 3025-3034.

10. Yigit H, Queenan AM, Anderson GJ, Domenech-Sanchez A, Biddle JW, Steward CD et al. Novel carbapenem-hydrolyzing beta-lactamase, KPC-1, from a carbapenem-resistant strain of Klebsiella pneumoniae. Antimicrob Agents Chemother 2001; 45 (4): 1151-1161.

11. Yong D, Toleman MA, Giske CG, Cho HS, Sundman K, Lee K et al. Characterization of a new metallo-beta-lactamase gene, bla(NDM-1), and a novel erythromycin esterase gene carried on a unique genetic structure in Klebsiella pneumoniae sequence type 14 from India. Antimicrob Agents Chemother 2009; 53 (12): 5046-5054.

12. Alcantar-Curiel MD, Rosales-Reyes R, Jarillo-Quijada MD, Gayosso-Vazquez C, Fernandez-Vazquez JL, Toledano-Tableros JE et al. Carbapenem-Resistant Acinetobacter baumannii in Three Tertiary Care Hospitals in Mexico: Virulence Profiles, Innate Immune Response and Clonal Dissemination. Front Microbiol 2019; 10: 2116.

13. Ambrosi C, Scribano D, Aleandri M, Zagaglia C, Di Francesco L, Putignani L et al. Acinetobacter baumannii Virulence Traits: A Comparative Study of a Novel Sequence Type with Other Italian Endemic International Clones. Front Microbiol 2017; 8: 1977.

14. Cerqueira GM, Peleg AY. Insights into Acinetobacter baumannii pathogenicity. IUBMB Life 2011; 63 (12): 1055-1060.

15. Almasaudi SB. Acinetobacter spp. as nosocomial pathogens: Epidemiology and resistance features. Saudi J Biol Sci 2018; 25 (3): 586-596.

16. Runnegar N, Sidjabat H, Goh HM, Nimmo GR, Schembri MA, Paterson DL. Molecular epidemiology of multidrug-resistant Acinetobacter baumannii in a single institution over a 10 -year period. J Clin Microbiol 2010; 48 (11): 4051-4056.

17. Weiner LM, Webb AK, Limbago B, Dudeck MA, Patel J, Kallen AJ et al. Antimicrobial-Resistant Pathogens Associated With HealthcareAssociated Infections: Summary of Data Reported to the National Healthcare Safety Network at the Centers for Disease Control and Prevention, 2011-2014. Infect Control Hosp Epidemiol 2016; 37 (11): 1288-1301.

18. Maroya Spalding Walters Comments to Author JEG, Bulens SN, Hancock EB, Phipps EC, Muleta D, Mounsey J, Kainer MA, Concannon C, Dumyati G, Bower C, Jacob J, Cassidy PM, Beldavs Z, Culbreath K, Phillips WE, Hardy DJ, Vargas RL, Oethinger M, Ansari U, Stanton R, Albrecht V, Halpin AL, Karlsson M, Rasheed JK, Kallen A. Carbapenem-Resistant Pseudomonas aeruginosa at US Emerging Infections Program Sites, 2015. Emerging Infectious Diseases 2019; 25.
19. Thaden JT, Park LP, Maskarinec SA, Ruffin F, Fowler VG, Jr, van Duin D. Results from a 13-Year Prospective Cohort Study Show Increased Mortality Associated with Bloodstream Infections Caused by Pseudomonas aeruginosa Compared to Other Bacteria. Antimicrob Agents Chemother 2017; 61 (6).

20. Castanheira M, Deshpande LM, Costello A, Davies TA, Jones RN. Epidemiology and carbapenem resistance mechanisms of carbapenemnon-susceptible Pseudomonas aeruginosa collected during 2009-11 in 14 European and Mediterranean countries. J Antimicrob Chemother 2014; 69 (7): 1804-1814.

21. Merck. MALDI-MS Analysis of Proteins 2020 [Available from: https://www.sigmaaldrich.com/life-science/proteomics/recombinant-protein-expression/protocols/maldims-analysis-of-proteins. html?gclid=Cj0KCQiAk53-BRD0ARIsAJuNhpvUwYCIMd5NxDbG8 w2Btapg0Mg0Ixf1ueMvjLxZgSP302NkYcR6CQaApLTEALw_wcB.

22. Disease ESoCMaI. EUCAST 2020 [Available from: https://www. eucast.org/clinical_breakpoints/.

23. Magiorakos AP, Srinivasan A, Carey RB, Carmeli Y, Falagas ME, Giske CG et al. Multidrug-resistant, extensively drug-resistant and pandrug-resistant bacteria: an international expert proposal for interim standard definitions for acquired resistance. Clin Microbiol Infect 2012; 18 (3): 268-281.

24. ECDC. Surveillance of antimicrobial resistance in Europe 2018. European Centre for Disease Prevention and Control; 2018, 18 Nov 2019.

25. Control ECfDPa. Antimicrobial resistance in the EU/EEA (EARSNet) - Annual Epidemiological Report for 2019. Surveillance report. European Centre for Disease Prevention and Control; 2020, 18 Nov 2020.

26. Peyclit L, Baron SA, Rolain JM. Drug Repurposing to Fight Colistin and Carbapenem-Resistant Bacteria. Front Cell Infect Microbiol 2019; 9: 193.

27. Ayerbe-Algaba R, Gil-Marques ML, Jimenez-Mejias ME, SanchezEncinales V, Parra-Millan R, Pachon-Ibanez ME et al. Synergistic Activity of Niclosamide in Combination With Colistin Against ColistinSusceptible and Colistin-Resistant Acinetobacter baumannii and Klebsiella pneumoniae. Front Cell Infect Microbiol 2018; 8: 348.

28. Hussein MH, Schneider EK, Elliott AG, Han M, Reyes-Ortega F, Morris F et al. From Breast Cancer to Antimicrobial: Combating Extremely Resistant Gram-Negative "Superbugs" Using Novel Combinations of Polymyxin B with Selective Estrogen Receptor Modulators. Microb Drug Resist 2017; 23 (5): 640-650.

29. Sun W, Sanderson PE, Zheng W. Drug combination therapy increases successful drug repositioning. Drug Discov Today 2016; 21 (7): 1189-1195.

30. Lazureanu V, Poroșnicu M, Gandac C, Moisil T, Bădițoiu L, Laza R et al. Infection with Acinetobacter baumannii in an intensive care unit in the Western part of Romania. BMC Infect Dis 2016; 16 Suppl 1 (Suppl 1): 95. 